\title{
Correction to: Outcomes of retesting in patients with previously uninformative cancer genetics evaluations
}

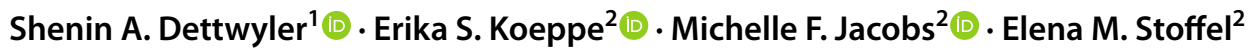

Published online: 28 October 2021

(c) Springer Nature B.V. 2021

Correction to: Familial Cancer

https://doi.org/10.1007/s10689-021-00276-8

In Table 4 of the article "Outcomes of retesting in patients with previously uninformative cancer genetics evaluations" Authored by "Shenin A. Dettwyler, Erika S. Koeppe,
Michelle F. Jacobs, Elena M. Stoffel, the data in the "Retest PV," "Retest VUS, "and "Retest Negative " columns do not align properly with the types of cancer in the first column. The correct Table 4 has been provided below.

The original article has been corrected.

The original article can be found online at https://doi.org/10.1007/ s10689-021-00276-8.

Shenin A. Dettwyler

shenin.dettwyler@nyulangone.org

1 The Pancreatic Cancer Center, NYU Langone Health, New York, NY, USA

2 Michigan Medicine Cancer Genetics Clinic, Ann Arbor, MI, USA 
Table 4 Cancer diagnoses and non-malignant manifestations in affected patients $(n=104)$

\begin{tabular}{|c|c|c|c|c|}
\hline & $\begin{array}{l}\text { Retest PV, } \\
n=21(20.2 \%)\end{array}$ & $\begin{array}{l}\text { Retest VUS, } \\
n=10(9.6 \%)\end{array}$ & $\begin{array}{l}\text { Retest negative, } \\
n=73(70.2 \%)\end{array}$ & $p^{\mathrm{a}}$ \\
\hline $\begin{array}{l}\text { Mean age at first diagnosis; years, } \\
\text { (range) } \\
\text { Total: } 42.5(2-73)\end{array}$ & $38.9(11-73)$ & $35.8(5-53)$ & $44.5(2-69)$ & 0.26 \\
\hline $\begin{array}{l}\geq 2 \text { primary diagnoses } \\
\text { Total: } n=42(40.4 \%)\end{array}$ & $8(19 \%)$ & $6(14.3 \%)$ & $28(66.7 \%)$ & 0.10 \\
\hline \multicolumn{5}{|c|}{ Cancer type/non-malignant manifestation ${ }^{\mathrm{b}, \mathrm{c}}$} \\
\hline Female breast $(n=42,40.4 \%)$ & $8(19 \%)$ & $3(7.1 \%)$ & $31(73.8 \%)$ & \\
\hline Melanoma $(n=27,26 \%)$ & $3(11.1 \%)$ & $2(7.4 \%)$ & $22(81.5 \%)$ & \\
\hline Colorectal $(n=21,20.2 \%)$ & $2(9.5 \%)$ & $3(14.3 \%)$ & $16(76.2 \%)$ & \\
\hline $\operatorname{Other}^{\mathrm{d}}(n=12,11.5 \%)$ & $4(33.3 \%)$ & $2(16.7 \%)$ & $5(41.7 \%)$ & \\
\hline Sarcoma $(n=10,9.6 \%)$ & $3(30 \%)$ & 0 & $7(70 \%)$ & \\
\hline PGL/PCC $(n=9,8.7 \%)$ & $2(22.2 \%)$ & $1(11.1 \%)$ & $6(66.7 \%)$ & \\
\hline Thyroid $(n=8,7.7 \%)$ & $1(12.5 \%)$ & $1(12.5 \%)$ & $6(75 \%)$ & \\
\hline Endometrial $(n=6,5.8 \%)$ & $2(33.3 \%)$ & 0 & $4(66.7 \%)$ & $>0.05$ \\
\hline Ovarian $(n=5,4.8 \%)$ & $2(40 \%)$ & $2(40 \%)$ & $1(20 \%)$ & \\
\hline $\operatorname{Polyposis}^{\mathrm{e}}(n=5,4.8 \%)$ & $3(60 \%)$ & 0 & $2(40 \%)$ & \\
\hline Genitourinary $(n=3,2.9 \%)$ & 0 & 0 & $3(100 \%)$ & \\
\hline Leukemia $(n=3,2.9 \%)$ & 0 & 0 & $3(100 \%)$ & \\
\hline $\operatorname{ACC}(n=3,2.9 \%)$ & 0 & 0 & $3(100 \%)$ & \\
\hline Oral SCC $(n=2,1.9 \%)$ & $2(100 \%)$ & 0 & 0 & \\
\hline Brain $(n=2,1.9 \%)$ & $1(50 \%)$ & $1(50 \%)$ & 0 & \\
\hline $\mathrm{HL}(n=2,1.9 \%)$ & $1(50 \%)$ & $1(50 \%)$ & 0 & \\
\hline Hypercalcemia $(n=2,1.9 \%)$ & $1(50 \%)$ & $1(50 \%)$ & 0 & \\
\hline Male breast $(n=2,1.9 \%)$ & 0 & 0 & $2(100 \%)$ & \\
\hline Prostate $(n=2,1.9 \%)$ & 0 & 0 & $2(100 \%)$ & \\
\hline Lung $(n=2,1.9 \%)$ & 0 & 0 & $2(100 \%)$ & \\
\hline
\end{tabular}

$A C C$, adrenocortical carcinoma; $H L$, Hodgkin's lymphoma; $P G L / P C C$, paraganglioma or pheochromocytoma; SCC, squamous cell carcinoma

${ }^{a}$ Compared informative (positive) results to uninformative results (VUS and negative results collectively)

${ }^{b}$ Includes non-malignant tumors associated with hereditary cancer predisposition syndromes and individuals with a clinical diagnosis of a hereditary cancer predisposition syndrome

${ }^{\mathrm{c}}$ Total $n>104$ and $>100 \%$, as $n=42$ had multiple primary cancers/non-malignant manifestations. Additional diagnoses of non-melanoma skin cancers alone were excluded

d“'Other” encompasses any cancer/non-malignant manifestation only observed once. Retest PV: gastrinoma, pancreatic neuroendocrine tumor, and primary hyperparathyroidism $(n=1$ each, all in same patient); peritoneal mesothelioma $(n=1)$. Retest VUS: ovarian granulosa cell tumor, retinal hemangioblastoma $(n=1$ each). Retest negative: bilateral acoustic neuromas, small bowel carcinoid, parotid gland tumor, sebaceous neoplasm; pancreatic ( $n=1$ each)

${ }^{\mathrm{e}}$ Clinical diagnosis of a hereditary polyposis syndrome. Retest PV: Familial Adenomatous Polyposis $(n=2)$ and Peutz-Jeghers syndrome $(n=1)$. Retest negative: Familial Adenomatous Polyposis and Juvenile Polyposis syndrome $(n=1$ each)

Publisher's Note Springer Nature remains neutral with regard to jurisdictional claims in published maps and institutional affiliations. 